\title{
Mind the Gap: Venous Thromboembolism Prophylaxis in Patients Hospitalized with Inflammatory Bowel Disease
}

\author{
John I. Allen
}

Published online: 21 November 2012

(c) Springer Science+Business Media New York 2012

As you board the train in London, a sign reminds you to "Mind the Gap" and not stumble between the train and boarding platform. Improving the quality of medical care will take countless efforts to "Mind the Gap" between care that could be and care that is. Bridge enough gaps and perhaps we can cross the "Quality Chasm" that the Institute of Medicine's 2001 report described [1].

In epidemiologic terms, the gap between best care and usual care is described by the terms "efficacy" and "effectiveness." A. L. Cochrane, the British epidemiologist, first articulated this difference in his classic book entitled Effectiveness and Efficiency: Random Reflections on Health Service, the textbook that launched evidencebased medicine [2]. According to Cochrane, efficacy refers to the best care achievable when provided under ideal (controlled) conditions. Effectiveness refers to care that is provided under average or usual conditions.

In this issue of Digestive Diseases and Sciences, a gap in care is highlighted for patients who are hospitalized with inflammatory bowel disease (IBD). Patients with IBD are at 3 - to 4-fold increased risk of both primary and recurrent venous thromboembolism (VTE) compared to people without IBD, VTE can occur at a younger age than expected and pulmonary embolism (PE) also appears at rates higher than in hospitalized patients without IBD [3-7]. VTE can occur in unusual locations including the cerebrovascular system, portal vein, mesenteric and retinal veins [3].

\section{J. I. Allen ( ()}

Minnesota Gastroenterology PA, 5705 Old Shakopee Road,

Bloomington, MN 55437, USA

e-mail: jallen@mngastro.com

\section{J. I. Allen}

University of Minnesota School of Medicine, Minneapolis, MN 55455, USA
In 2003 there were approximately 38 million discharges from US acute care hospitals [8]. Fifty-one percent of the 15 million patients discharged from medical services were judged to be at increased risk for VTE according to criteria of the American College of Chest Physicians (ACCP) [9]. VTE contributes to over 100,000 deaths each year and is one of the Agency for Healthcare Research and Quality's (AHRQ) key strategies for improving patient safety [10]. The following is taken from the ACCP 2012 VTE guidelines:

For acutely ill hospitalized medical patients at increased risk of thrombosis, we recommend anticoagulant thromboprophylaxis with low-molecularweight heparin (LMWH), low-dose unfractionated heparin... or fondaparinux (Grade 1B).

Sam et al. [11] at Mount Sinai Hospital Centre for Inflammatory Bowel Disease (Toronto) studied physicians' perceptions of risks and practices in VTE prophylaxis in IBD patients. They surveyed all members of the American Gastroenterological Association (AGA) who were affiliated with the Immunology, Microbiology and IBD section. Practicing clinicians who had cared for IBD patients were invited by email to participate in a survey of their perceptions and practices concerning VTE prophylaxis. A total of 174 gastroenterologists responded to the email invitation and 135 completed the survey (14\% of the 1,248 members of the AGA). Most (77\%) practiced in academic medical centers and had over 14 years (mean) of practice experience; $41 \%$ had $>50 \%$ IBD patients in their practice signifying the expertise of this sample. Most (84\%) reported having had IBD patients develop VTE and almost all agreed that hospitalized IBD patients have a higher risk of VTE compared to non-IBD patients. Over half knew that VTE in IBD patients was associated with excess mortality. 
Table 1 Examples of quality improvement initiatives and infrastructure

\begin{tabular}{|c|c|}
\hline Objective & Description of example \\
\hline Internal quality improvement program & $\begin{array}{l}\text { Often in response to patient experience, task force examines } \\
\text { literature, discussion among providers }\end{array}$ \\
\hline $\begin{array}{l}\text { Internal process re-engineering from bedside to system } \\
\text { wide change }\end{array}$ & $\begin{array}{l}\text { Institute for healthcare improvement framework for spread (see } \\
\text { Ref. [18]) }\end{array}$ \\
\hline $\begin{array}{l}\text { Increase awareness among providers at point of care automate or } \\
\text { mandate actions when deemed necessary }\end{array}$ & $\begin{array}{l}\text { Clinical decision support tools (Red Flag Alerts, algorithms } \\
\text { embedded in the electronic medical record), standard order sets } \\
\text { as default with over-ride capability }\end{array}$ \\
\hline Outcome registries to track performance & $\begin{array}{l}\text { Digestive Health Outcomes Registry (AGA), National Surgical } \\
\text { Quality Improvement Program (NSQIP) of the American } \\
\text { College of Surgeons }\end{array}$ \\
\hline Recognition programs for high quality performance & $\begin{array}{l}\text { Digestive Health Recognition Program (AGA), Crohn's and } \\
\text { Colitis Foundation of America (CCFA) }\end{array}$ \\
\hline Financial or reimbursement alteration public reporting & $\begin{array}{l}\text { Hospital Compare, Physician Quality Reporting System, Physician } \\
\text { Compare }\end{array}$ \\
\hline
\end{tabular}

The column on the left (objectives) lists the reasons and goals of quality improvement initiatives. Examples of such initiatives are provided on the right column

AGA American Gastroenterological Association

Twenty-six percent did not routinely assess their patients for signs or symptoms of VTE on admission. As noted in the AHRQ presentation cited above [9], public reporting of physician VTE assessment is coming (for now confined to joint replacement patients) and soon the Centers for Medicare and Medicaid (CMS) will not pay for hospitalacquired complications including VTE. Anticipate more "sticks" to appear since VTE prophylaxis is seen as a highly effective maneuver to enhance patient safety.

Forty-seven percent of these physicians self-reported administering VTE prophylaxis all of the time, $76 \%$ at least half the time and $14 \%$ never administer prophylaxis. One in five physicians surveyed believed there was insufficient evidence that VTE prophylaxis was either effective or safe enough to warrant routine administration. VTE prophylaxis was deemed unnecessary in young ( $<40$ years) and ambulatory IBD patients in 8 and $24 \%$, respectively. There was conflicting opinion about whether rectal bleeding was a contra-indication to pharmacological prophylaxis. At least one randomized controlled study failed to show a significant difference in all-cause mortality between hospitalized patients at increased risk for VTE provided graduated compression stockings versus low-molecularweight heparin, suggesting a good alternative to actively bleeding IBD patients [12]. Even aspirin has been demonstrated to be useful to prevent recurrent VTE [13]. Other excellent VTE management reviews and step-by-step improvement strategies have been published [14-18].

Given the variation in practice among experienced IBD physicians, one can only speculate about VTE prophylaxis among physicians who care for hospitalized IBD patients less frequently. What might be done to close this gap? Quality improvement efforts can involve focused education, publication of evidence-based guidelines, internal process improvement, outcome registries, recognition programs and financial incentives (or penalties). Examples of improvement initiatives are outlined in Table 1.

Transforming a health care system to deliver consistently high quality care for clinical service lines or a disease process is difficult. Such efforts begin with the development of an organizational culture of quality and recruitment of committed clinical leaders. Foundational knowledge about achieving high-value, cost-conscious health care is essential for leaders of any improvement effort [19]. The Institute for Healthcare Improvement (IHI) has long been a source of practical advice about implementation of change from bedside to system-wide [20]. IHI is an independent not-forprofit organization based in Cambridge, Massachusetts focused on improving health care delivery. An open-source interactive "Improvement Map" is available to help initiate a change strategy and track progress [21]. One portion of the multiple processes targeted for improvement is VTE prophylaxis.

Nationally, we are transitioning from the use of electronic medical records that merely record data to informational tools that drive behavior change and care improvement. One method used to enhance consistent care around a single disease or intervention is through the use of Clinical Decision Support Tools such as reviewed in VTE management [22].

Outcome registries are useful to identify specific interventions or outcomes that are deemed to be of highest value to patient care. At least three outcomes registries are available to track IBD care in children [23], adults [24] and surgical patients [25]. Goals of these emerging registries, in addition to demonstrating improvement in patient care, 
include helping practices become recognized as centers of excellence for IBD care either by commercial or government payers or the lay public.

Two national recognition programs focused on IBD care now exist. The AGA has partnered with Bridges to Excellence (BTE) to develop a Digestive Health Recognition Program (DHRP) [26]. DHRP begins with practitioners extracting specific data elements from 24 consecutive IBD patient charts and filling out a template that is sent to an adjudicating entity. If sufficient points are achieved (based on meeting performance standards) a 2-year recognition status is conferred by BTE and AGA jointly. This is the first gastroenterology-specific BTE program. Other specialty programs recognized by numerous commercial payers are used to differentiate reimbursement. Crohn's and Colitis Foundation of America (CCFA) has developed criteria that characterize a high quality IBD practice using measures similar to those of the AGA. Despite the demonstrated relationship between IBD and increased risk for VTE, routine assessment of hospitalized IBD for VTE risk is not yet a performance measure in current registries or recognition programs. As programs develop and change, consideration for such a measure would be recommended.

Healthcare in the United States is beginning to transform from volume-based (fee for service) to value-based payments where financial risk is tied to health outcomes or patient experience. The Hospital Compare and Physician Compare programs of CMS are mandated by law to begin differentiating reimbursement to hospitals and providers respectively based on achieving high quality outcomes or avoiding adverse events. Avoidable complications such as VTE or PE are tied to diminished financial reimbursement [26]. The Physician Quality Reporting System (PQRS) from CMS is a program whereby providers report on specific performance measures that can either be individual measures or group measures. An IBD group was developed by a joint task force from the AGA and CCFA and now is part of the 2012 PQRS (measures 226 plus 269-276). These can only be reported through a registry such as AGA's DHOR.

Articles such as the one by Sam et al. [27] illustrate the challenge we have to improve care, gain consensus on best practice and develop an infrastructure to drive behavior change and measure performance. The "Quality Agenda" of the United States has been clearly articulated by the National Quality Forum in its 2012 report to Congress. A "Quality Care Primer" for gastroenterologists has been published [28] with multiple opportunities to measure and report on performance improvement now in existence (cited above). It is now up to us collectively to "Mind the Gap" and cross the "Quality Chasm."
Conflict of interest None.

\section{References}

1. Institute of Medicine. Crossing the quality chasm: a new health system for the 21st century. Washington, DC: National Academy Press; 2001.

2. Cochrane AL. Effectiveness and Efficiency. Random Reflections on Health Services. London: Nuffield Provincial Hospitals Trust, 1972. (Reprinted in 1989 in association with the BMJ, Reprinted in 1999 for Nuffield Trust by the Royal Society of Medicine Press, London (ISBN 1-85315-394-X), pp 26-45.

3. Novacek G, Weltermann A, Sobala A, et al. Inflammatory bowel disease is a risk factor for recurrent venous thromboembolism. Gastroenterology. 2010;139:779-787.

4. Talbot RW, Heppell J, Dozois RR, et al. Vascular complications of inflammatory bowel disease. Mayo Clin Proc. 1986;61: 140-145.

5. Bernstein CN, Blanchard JF, Houston DS, et al. The incidence of deep venous thrombosis and pulmonary embolism among patients with inflammatory bowel disease: a population-based cohort study. Thromb Haemost. 2001;85:430-434.

6. Danese S, Papa A, Saibeni S, et al. Inflammation and coagulation in inflammatory bowel disease: the clot thickens. Am J Gastroenterol. 2007;102:174-186.

7. Grip O, Svensson PJ, Lindgren S. Inflammatory bowel disease promotes venous thrombosis earlier in life. Scand J Gastroenterol. 2000;35:619-623.

8. Anderson FA, Zayaruzny M, Heit JA, Fidan D, Cohen AT. Estimated annual numbers of US acute-care hospital patients at risk for venous thromboembolism. Am J Hematol. 2007;82(9): 777.

9. Kahn SR, Lim W, Dunn AS, et al. Antithrombotic therapy and prevention of thrombosis, 9th ed: American College of Chest Physicians evidence-based clinical practice guidelines. Chest. 2012;141(suppl. 2):11-2296.

10. AHRQ. Venous thromboembolism (VTE) prevention in the hospital. http://www.ahrq.gov/qual/vtepresentation/maynardtxt.htm. Accessed October 15, 2012.

11. Sam JJ, Bernstein CN, Razik R, Thanabalan R, Nguyen GC. PhysiciansGú $\varnothing$ perceptions of risks and practices in venous thromboembolism prophylaxis in inflammatory bowel disease. Dig Dis Sci. (Epub ahead of print). doi:10.1007/s10620-0122435-6.

12. Kakkar AK, Cimminiello C, Goldhaber SZ, Rarakh R, Wang C, et al. Low-molecular-weight heparin and mortality in acutely ill medical patients. New Eng J Med. 2011;365:2463-2477.

13. Becattini C, Agnelli G, Schenone A, et al. Aspirin for preventing the recurrence of venous thromboembolism. New Eng J Med. 2012;366:1959-1967.

14. Moheimani F, Jackson DE. Venous thromboembolism: classification, risk factors, diagnosis, and management. Intern Sch Res Netw Hematol. 2011;2011:124610-124617.

15. Abdel-Razeq H. Venous thromboembolism prophylaxis for hospitalized medical patients, current status and strategies to improve. Ann Thorac Med. 2012;5(4):195-200.

16. AHRQ. Preventing hospital-acquired venous thromboembolism.Appendix A: talking points to attract administration support for venous thromboembolism prevention programs. http://www.ahrq. gov/qual/vtguide/vtguideapa.htm. Accessed October 15, 2012.

17. Franci CW. Prophylaxis for thromboembolism in hospitalized medical patients. New Eng J Med. 2007;356:1438-1444.

18. Agnelli G, Becattini C. Acute pulmonary embolism. New Eng J Med. 2010;363:266-274. 
19. Owens DK, Qaseem A, Chou R, Shekelle P, et al. High-value, cost-conscious health care: concepts for clinicians to evaluate the benefits, harms and costs of medical interventions. Ann Intern Med. 2011;154:174-180.

20. Massoud MR, Nielsen GA, Nolan K, Schall MW, Sevin C. A Framework for Spread: From Local Improvements to SystemWide Change. IHI Innovation Series white paper. Cambridge, MA: Institute for Healthcare Improvement; 2006. (Available on www.IHI.org).

21. IHI. Materials. http://www.ihi.org/offerings/Initiatives/Improve maphospitals/Pages/Materials.aspx. Accessed October 15, 2012.

22. Hogg K, Wells PS, Gandara E. The diagnosis of venous thromboembolism. Semin Thromb Hemost. 2012; Epub. 10/03/2012.

23. Improve Care Now. https://improvecarenow.org. Accessed October $15,2012$.

24. AGA. AGA Digestive Health Outcomes Registry®. http://www. gastro.org/practice/digestive-health-outcomes-registry. Accessed October 15, 2012.
25. Wallaert JB, De Martino RR, Marsicovetere PS, et al. Venous thromboembolism after surgery for inflammatory bowel disease: are there modifiable risk facotrs $>$ Data from ACS NSQIP. Dis Colon Rectum. 2012;55(11):1138-1144.

26. USDHHS. Hospital compare. http://hospitalcompare.hhs.gov. Accessed October 16, 2012.

27. National Quality Forum. Changing Healthcare by the numbers; 2012 NQF Report to Congress. http://www.qualityforum.org/ Publications/2012/03/2012_NQF_Report_to_Congress.aspx. Accessed October 16, 2012.

28. Kappelman MD, Dorn SD, Peterson E, Runge T, Allen JI. Quality of care for gastrointestinal conditions: a primer for gastroenterologists. Amer J Gastroenterol. 2011;106:1182-1187. 